\title{
Caracterización del hábitat de peces nativos en el río San Pedro (cuenca del rio Valdivia, Chile)
}

\section{Habitat characterization of native fish in the San Pedro River (Valdivia River basin, Chile)}

\author{
Alex Garcia ${ }^{1 *}$, Jorge González ${ }^{1} \&$ Evelyn Habit ${ }^{1}$ \\ ${ }^{1}$ Centro de Ciencias Ambientales EULA-Chile, Universidad de Concepción, Barrio Universitario s/n, casilla 160-C, Concepción, \\ Chile \\ *Email: alexgarcia@udec.cl
}

\begin{abstract}
RESUMEN
En sistemas fluviales, las comunidades biológicas están estructuradas principalmente por el hábitat físico. Conocer el tipo de hábitat que ocupa cada especie a lo largo de su vida es fundamental para comprender su ecología en sistemas naturales y definir estrategias de conservación en sistemas alterados. Por ello, aquí caracterizamos el uso de hábitat de 12 especies de peces nativos del río San Pedro. Se capturaron 37334 individuos de las 13 especies nativas presentes en el río. Los hábitats más muestreados y con mayores capturas totales fueron pozas someras y rápidos someros. Las especies más abundantes fueron Percilia gillissi, Galaxias maculatus, Galaxias platei y Trichomycterus areolatus. Se observa un uso diferenciado del hábitat por especie, en que los Galáxidos son más abundantes en zonas de baja velocidad (pozas) y los Siluriformes en zonas de alta velocidad (rápidos). Además, reconocemos el uso diferenciado del hábitat con la talla en Aterinópsidos y Percichthys trucha, en que los adultos ocupan principalmente los ambientes lacustres y sus juveniles las zonas fluviales ribereñas.
\end{abstract}

Palabras clave: Hábitat, peces nativos, río San Pedro, Chile

\begin{abstract}
In fluvial systems, the biological communities are mainly structured by the physical habitat. Knowing the habitat that each species use throughout its life is fundamental to understand its ecology in natural systems and to define conservation strategies in altered systems. For that reason, here we characterize the habitat use of 12 native fish species of the San Pedro River. 37344 individuals of the 13 native species occurring in the river were caught. Shallow ponds and shallow rapids were the most sampled habitats and had the largest captures. The most abundant species were Percilia gillissi, Galaxias maculatus, Galaxias. platei and Trichomycterus areolatus. A differentiated habitat use per species is notices, where the Galaxiids are more abundant in areas of slow velocity (ponds) and the Silurids in areas of high velocity (riffles). Moreover, we recognized a differentiated habitat use based on the size of Atherinopsidae and Percichthys trucha, where adults mainly occur in the lake and their juveniles occur in the borders of fluvial zones.
\end{abstract}

KeYwords: Habitat, native fish, San Pedro River, Chile

\section{INTRODUCCIÓN}

La estructura y funcionamiento de las comunidades fluviales están fuertemente determinadas por la organización, estructura y dinámica del hábitat físico del cauce (Vannote et al. 1980). Éste se presenta como un mosaico de parches de hábitat que varían en cantidad y calidad a lo largo del cauce (Skyfield \& Grossman 2008), y que influyen en la distribución y el uso de hábitat de los peces (Grossman \& Freeman 1987; Freeman \& Grossman 1993). Tales parches de hábitat, también descritos como unidades geomorfológicas de cauce (Hawkins et al. 1993) o mesohábitats (Tickner et al. 2000; Parasiewicz 2001), son unidades físicas homogéneas que se pueden describir por variables físicas como la velocidad (V) y la profundidad (Y) de la corriente, el tipo de substrato y combinaciones de éstas como el número de Froude $\left(\mathrm{Fr}=3,13 \cdot \mathrm{V} \cdot \mathrm{Y}^{-0,5}\right)$ (Jowett 1993, Clifford et al. 2006). Los requerimientos de hábitat de los peces presentan cambios ontogénicos, así como sus dietas, posición jerárquica y trófica (Werner \& Hal 1976; Werner \& Gill 1984). Por lo tanto, el conocimiento del uso de hábitat de las especies de peces nativos es fundamental para entender 
su ecología en sistemas naturales y para establecer medidas de manejo y conservación. En Chile existen pocos estudios sobre uso de hábitat de peces nativos, y por lo general sólo abarcan tramos de río (Arratia 1983; Habit 2005; Penaluna et al. 2009; García et al. 2011; Vargas et al. 2010). El objetivo de este trabajo es describir el uso de hábitat ribereño de 12 especies de peces nativos que se encuentran a lo largo del río San Pedro (Región de los Ríos, Chile).

\section{MATERIALES Y MÉTODOS}

\section{Zona De Estudio}

El estudio fue realizado en el río San Pedro, en la Cuenca del río Valdivia $\left(10275 \mathrm{~km}^{2}\right)$. El río San Pedro, de $38 \mathrm{~km}$ de longitud, se ubica en la zona media de la cuenca y comprende desde el desagüe del lago Riñihue hasta la confluencia con el río Quinchilcas (Figura 1). El régimen de caudales es controlado por la presencia de los lagos Araucanos (sensu Thomasson 1963), con un caudal medio de $404 \mathrm{~m}^{3} / \mathrm{s}$. La heterogeneidad de hábitats en el río San Pedro se presenta tanto espacial como temporalmente, incluyendo ambientes terrestres en períodos de crecidas. El río fluye relativamente confinado en la parte alta, con un cauce profundo donde abundan ambientes de velocidad baja. En la parte media, el río fluye encajonado en un valle estrecho, con pendiente alta y abundancia de rápidos (velocidad alta). En la parte baja se observan secuencias de pozas y rápidos y una mayor interacción del río con la planicie de inundación.
CARACTERIZACIÓN DE PARCHES DE HÁBITAT

Se muestreó un total de 22 sitios para caracterizar cinco tipos de hábitat ribereño y dos en el lago Riñihue durante los primeros meses de campañas de muestreo de peces. Se midieron velocidad y profundidad del escurrimiento en varios puntos de cada sitio, considerando al menos tres puntos en cada parche de hábitat y el tamaño medio del substrato se categorizó visualmente como fino (fango), arena $(<0,8$ $\mathrm{cm})$, grava $(0,8-20 \mathrm{~cm})$, bolones $(>20 \mathrm{~cm})$. La velocidad $\mathrm{y}$ profundidad fueron medidos con un correntómetro Gurley modelo Pygmy modelo 625. Los parches de hábitat presentes en estos sitios fueron definidos subjetivamente por el grupo de muestreo de peces. El criterio para la clasificación de hábitats ribereños consideró el primer nivel de clasificación (por velocidad) de Hawkins et al. (1993) y luego los agrupó como somero o profundo: i) poza somera: flujo lento con superficie del agua lisa, profundidad baja y substrato fino (arena, limo o arcilla) o grueso (grava, bolones) con presencia de fino; ii) poza profunda: flujo lento con superficie del agua lisa, profundidad media (vadeable) y substrato fino o grueso con presencia de fino; iii) rápido somero: flujo rápido con superficie del agua ondulada o rota, profundidad baja y substrato grueso con poca presencia de fino; iv) flujo rápido con superficie del agua ondulada o rota, profundidad media (vadeable) y poca presencia de fino. Además, para describir el uso de hábitat de algunas especies/estadios vitales particulares se definió los hábitats de: v) poza temporal: flujo lento sobre la planicie de inundación (en cualquier tipo de substrato o vegetación), vi) playa: zona litoral vadeable del lago Riñihue, y vii) lago: zona profunda del lago Riñihue.

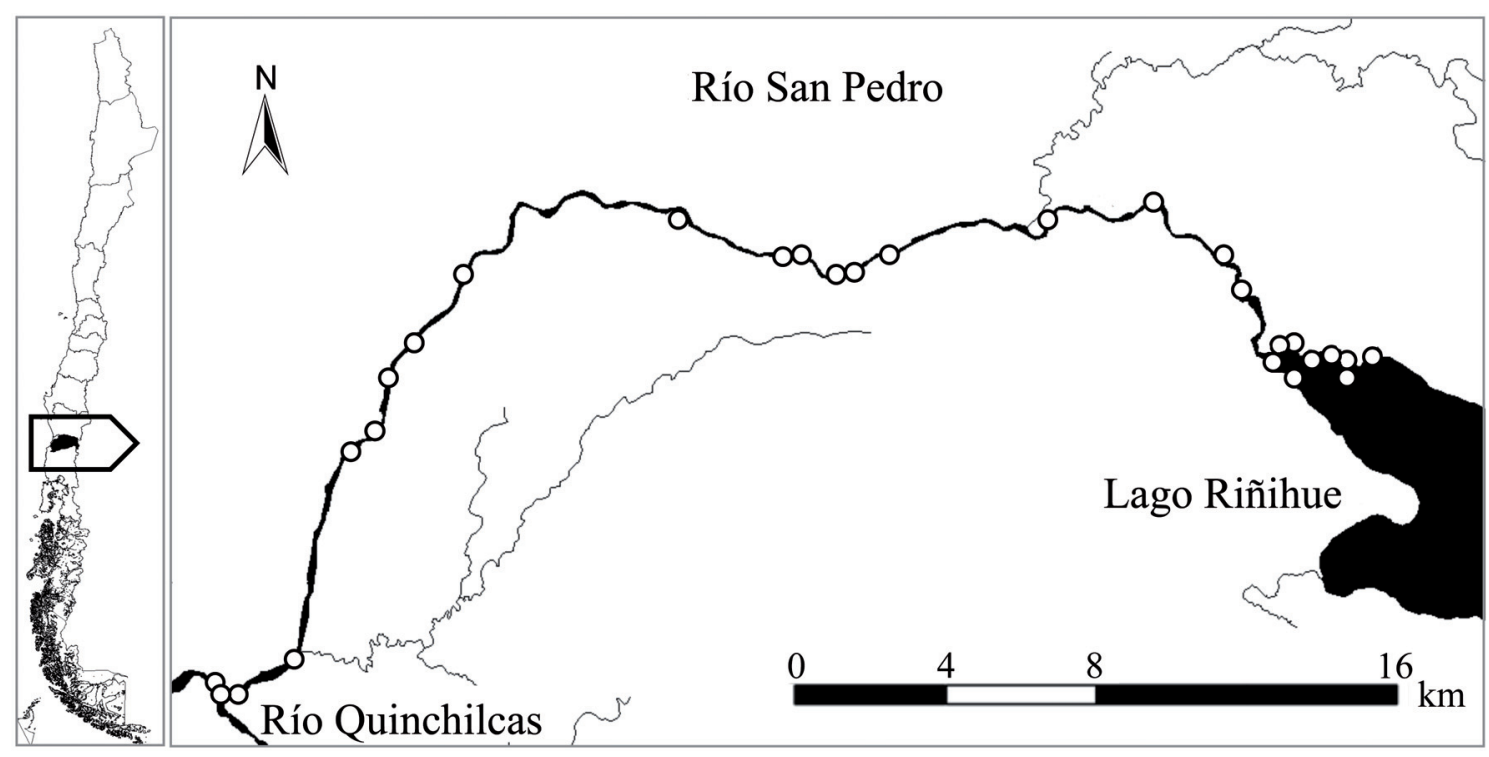

Figura 1. Zona de estudio. Río San Pedro en la cuenca del río Valdivia. Los círculos indican los sitios de muestreo.

Figure 1. Study site. San Pedro River in the Valdivia River Basin. Sampling sites are indicated with circles. 
Un análisis de varianza (ANOVA) univariado mostró que las variables hidráulicas velocidad, profundidad de escurrimiento y número de Froude presentan diferencias significativas entre los hábitats poza somera, poza profunda, rápido somero y rápido profundo (Tabla 1). En esta clasificación, los hábitat de poza caen dentro de los 'pool' descritos por Jowett (1993), y los rápidos están en la zona de 'runs'. Un test de Tukey de comparación pareada de las medias de las variables hidráulicas muestra diferencias significativas $(\mathrm{P}<0,01$ en todos los casos) entre los hábitats de poza (somera y profunda) con los rápidos (somero y profundo) para la variable velocidad; y muestra diferencias entre los hábitats someros y profundos para la variable profundidad. Los hábitats de rápido profundo mostraron diferencias significativas con rápido somero para las variables profundidad y número de Froude; mientras que las pozas someras y profundas mostraron diferencias significativas sólo para profundidad.

\section{Muestreo de Peces}

Un total de 465 eventos de pesca fueron realizados durante 289 días, desde octubre de 2005 hasta julio de 2008. Se pescó mayoritariamente de día. Se muestrearon 28 sitios (Figura 1) con pesca eléctrica (LR-24 Electrofisher, Smith Root, Inc.), redes de arrastre ( $2 \mathrm{~mm}$ de trama) y redes agalleras pelágicas multitrama ( 25 y $65 \mathrm{~mm}$ de trama). Los muestreos con pesca eléctrica fueron realizados en el río con un mínimo de tres pescadores, durante 45 minutos y cubriendo $200 \mathrm{~m}^{2}$ por hábitat, en promedio. Se realizaron prospecciones con redes en la zona litoral y pelágica del lago Riñihue. Los ejemplares capturados fueron mantenidos en baldes con aireación continua, luego fueron sedados (BZ-20), identificados a nivel de especie, medidos (longitud estándar), pesados y devueltos al lugar donde fueron capturados. En cada evento de pesca se registró el nombre del sitio, la fecha, el tipo de hábitat del parche, variables físicas (velocidad, profundidad del escurrimiento y tipo de substrato) y la cantidad de individuos colectados de cada especie (con talla y peso de cada individuo).

\section{AnÁlisis DE DATOS}

Para cuantificar el uso de hábitat por especie se determinó la captura de peces por unidad de esfuerzo (CPUE) en cada evento de pesca eléctrica. Los valores mayores de CPUE indican más uso del hábitat. CPUE fue calculada como la abundancia de peces (por especie) sobre el tiempo de pesca y el área del hábitat muestreado. Además, se determinó la longitud promedio de todos los individuos capturados, por especie, en cada hábitat, para determinar cambios ontogénicos en el uso de hábitat. Aplochiton zebra no fue considerado en este análisis debido al bajo número de individuos colectados.

A nivel comunitario, se realizó un análisis de similitud ANOSIM (Clarke \& Gorley 2005), que trabaja sobre una matriz de similitud, para evaluar el grado de disimilitud entre los hábitats basado en la abundancia de individuos por especie. La matriz de similitud se calculó con el índice de Bray-Curtis sobre la matriz de CPUE por sitio y por especie, estandarizada y transformada $\left({ }^{4} \sqrt{ }\right)$. Luego, se identificaron las especies que caracterizan cada tipo de hábitat con la rutina SIMPER. Dado que Percilia gillisi Girard, 1854 fue la especie más abundante y ocupó indistintamente tanto rápidos como pozas, fue eliminada de este análisis. De este modo, los resultados de SIMPER indican sólo las especies que hacen uso diferenciado de hábitats. Los análisis comunitarios fueron realizados con el programa PRIMER V6 (Clarke \& Gorley 2005).

TABLA 1: Características hidráulicas (promedio $\pm \mathrm{DE}$ ) de los lugares clasificados como poza somera, poza profunda, rápido somero y rápido profundo y el estadístico $F$ de ANOVA para la prueba de igualdad de las medias. $* \mathrm{P}<0,001$.

TABLE 1: Hydraulic characteristics (mean $\pm \mathrm{SD}$ ) of the locations classified as poza somera, poza profunda, rápido somero y rápido profundo and the ANOVA $F$-statistic for testing the probability that the means are equal. ${ }^{*} \mathrm{P}<0,001$.

\begin{tabular}{lccccc}
\hline & $\begin{array}{c}\text { Poza somera } \\
\mathrm{n}=57\end{array}$ & $\begin{array}{c}\text { Poza profunda } \\
\mathrm{n}=27\end{array}$ & $\begin{array}{c}\text { Rápido somero } \\
\mathrm{n}=60\end{array}$ & $\begin{array}{c}\text { Rápido } \\
\text { profundo } \\
\mathrm{n}=101\end{array}$ & $F$-statistic \\
\hline Profundidad (m) & $0,30 \pm 0,12$ & $0,76 \pm 0,18$ & $0,65 \pm 0,24$ & $0,30 \pm 0,11$ & $122,07^{*}$ \\
Velocidad (m/s) & $0,05 \pm 0,05$ & $0,04 \pm 0,04$ & $0,39 \pm 0,31$ & $0,40 \pm 0,25$ & $47,14^{*}$ \\
Fr & $0,02 \pm 0,02$ & $0,01 \pm 0,01$ & $0,11 \pm 0,08$ & $0,17 \pm 0,11$ & $51,84^{*}$ \\
\hline
\end{tabular}


Hábitat peces San Pedro: Alex García ET AL.

TABLA 2: Capturas totales por especie en cada tipo de hábitat.

TABLE 2: Total captures by species at each habitat type.

\begin{tabular}{|c|c|c|c|c|c|c|c|c|}
\hline Especies & Lago & Playa & PS & PP & PT & RS & $\mathbf{R P}$ & Total \\
\hline Galaxias maculatus & & 2681 & 4262 & 97 & 956 & 600 & 43 & 8639 \\
\hline Galaxias platei & & 176 & 3703 & 207 & 623 & 184 & 24 & 4917 \\
\hline Aplochiton taeniatus & & 26 & 198 & 7 & 3 & 93 & 9 & 336 \\
\hline Aplochiton zebra & & & 14 & & 1 & 3 & & 18 \\
\hline Brachygalaxias bullocki & & & 483 & & 68 & 81 & 4 & 636 \\
\hline Percilia gillissi & & 222 & 4512 & 194 & 239 & 2995 & 593 & 8755 \\
\hline Percichthys trucha & 5 & 18 & 515 & 21 & 53 & 174 & 28 & 814 \\
\hline Basilichthys australis & 8 & 334 & 1396 & 90 & 1746 & 225 & 14 & 3813 \\
\hline Odontesthes mauleanum & 5 & 75 & 6 & 2 & 3 & 3 & 1 & 95 \\
\hline Diplomystes camposensis & & & 406 & & 13 & 733 & 235 & 1387 \\
\hline Trichomycterus areolatus & & 27 & 2086 & 11 & 172 & 2254 & 287 & 4837 \\
\hline \multirow{2}{*}{$\begin{array}{l}\text { Cheirodon australe } \\
\text { Geotria australis }\end{array}$} & & 22 & 1267 & 53 & 568 & 91 & 6 & 2007 \\
\hline & & & 786 & 7 & 140 & 112 & 32 & 1077 \\
\hline Total & 18 & 3581 & 19634 & 689 & 4585 & 7548 & 1276 & \\
\hline
\end{tabular}

PS: poza somera, PP: poza profunda, PT: poza temporal, RS: rápido somero, RP: rápido profundo

PS: shallow pond, PP: deep pond, PT: temporal pond, RS: shallow rapid, RP: deep rapid

TABLA 3: Hábitats a nivel comunitario. Se indica los porcentajes de similitud para hábitats de poza y rápido. La contribución por especie a cada tipo de hábitat se indica hasta un $80 \%$.

TABLE 3: Habitats at a community level. The similarity percentage for pool and riffle type of habitats are shown. The contribution per species to each habitat type is shown up to $80 \%$.

\begin{tabular}{|c|c|c|c|}
\hline & $\begin{array}{l}\text { Promedio } \\
\text { CPUE* }\end{array}$ & $\begin{array}{c}\text { Contribución } \\
(\%)\end{array}$ & $\begin{array}{c}\text { Contribución acumulada } \\
(\%)\end{array}$ \\
\hline $\begin{array}{l}\text { Pozas }(\mathbf{4 6 , 4 \% )} \\
\text { Galaxias maculatus }\end{array}$ & 20,5 & 26,9 & 26,9 \\
\hline Galaxias platei & 18,5 & 24,4 & 51,3 \\
\hline Trichomycterus areolatus & 15,4 & 17,3 & 68,6 \\
\hline Cheirodon australe & 10,2 & 9,2 & 77,8 \\
\hline Percichthys trucha & 8,4 & 6,8 & 84,6 \\
\hline $\begin{array}{l}\text { Rápidos }(\mathbf{4 1}, \mathbf{8 \%}) \\
\text { Trichomycterus areolatus }\end{array}$ & 34,3 & 52,8 & 52,8 \\
\hline Diplomystes camposensis & 19,2 & 20,9 & 73,7 \\
\hline Galaxias maculatus & 14,3 & 13,5 & 87,2 \\
\hline$\%$ similitud entre ensambles & & 34,9 & \\
\hline
\end{tabular}

* CPUE transformada $(\sqrt[4]{ })$ y estandarizada. 


\section{RESULTADOS}

En este estudio se capturaron 37334 individuos de 13 especies nativas (Tabla 2). Pozas y rápidos someros fueron los hábitats con mayor numero de eventos de pesca y capturas totales (Tabla 2). Las especies más abundantes (capturas totales) fueron P. gillissi, Galaxias maculatus (Jenyns 1842), G. platei Steindachner, 1898 y Trichomycterus areolatus Valenciennes, 1846 (Tabla 2).

En la Figura 3 se observa los resultados de abundancia (como CPUE) y talla promedio para cada especie en cada uno de los hábitats muestreados. Los Galáxidos, G. maculatus y $G$. platei mostraron mayores CPUE en hábitats de poza (poza somera PS, poza profunda PP, y poza temporal PT). Por otro lado, las menores tallas (longitud estándar promedio) para ambas especies se encontraron en playa de lago y las mayores en rápido profundo. Aplochiton taeniatus fue más abundante en rápido somero, con individuos de menor talla en hábitats de playa y de tallas mayores en hábitats de rápido profundo. No se aprecian diferencias en abundancia y talla por tipo de hábitat para Brachygalaxias bullocki. Las mayores abundancias de P. gillissi y Percichthys trucha se dieron en rápido somero. Percichthys trucha presentó las mayores tallas en el lago Riñihue, mientras que en ambientes de río ambas especies tienden a presentar mayores tallas en
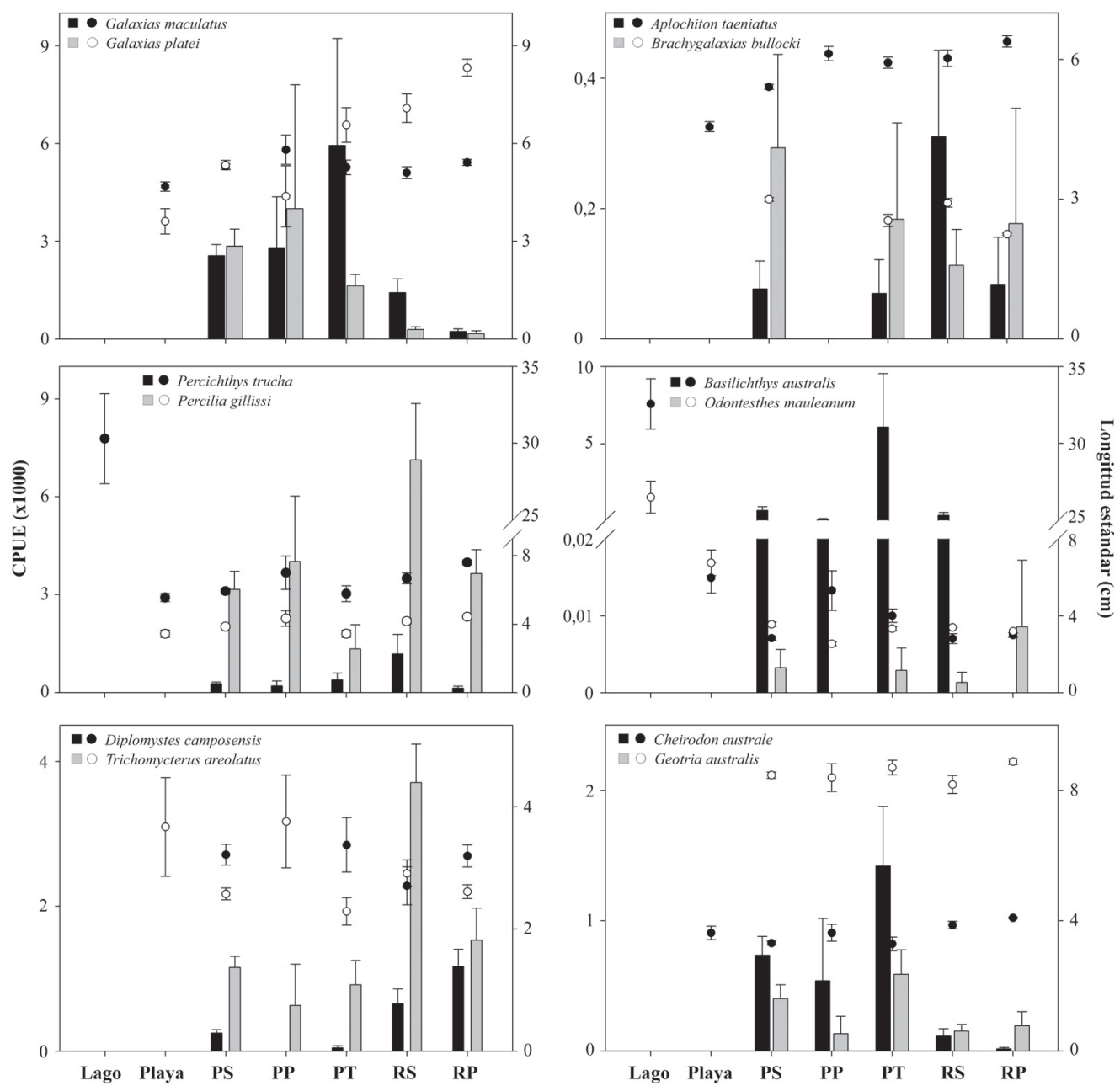

Hábitats

Figura 2. Uso de hábitat de peces nativos, expresado como CPUE (barras, +-error estándar) y longitud estándar (puntos, terror estándar). PS: poza somera, PP: poza profunda, PT: poza temporal, RS: rápido somero, RP: rápido profundo

FigURE 2. Habitat use of native fish shown as CPUE (bars, +standard error) and standard length (dots, \pm standard error). PS: shallow pond, PP: deep pond, PT: temporal pond, RS: shallow rapid, RP: deep rapid. 
hábitats profundos. Basilichthys australis presentó mayores abundancias que Odontesthes mauleanum principalmente en ambientes de rápido somero. Las mayores tallas para ambas especies se dieron en el lago Riñihue y fueron menores en hábitats rápidos. Los bagres Diplomystes camposensis y $T$. areolatus presentaron mayor abundancia en hábitats rápidos. Trichomycterus areolatus presentó tallas menores en poza temporal, mientras que D. camposensis presentó tallas menores en rápido somero. Las especies Cheirodon australe y Geotria australis presentaron mayores abundancias en hábitats de poza, sin una diferenciación por tallas entre hábitats.

Los análisis comunitarios basados en abundancia (CPUE) por especies, muestran diferencias significativas entre los hábitats agrupados por velocidad como rápidos (RS, RP) y pozas (PS, PT, PP)(ANOSIM, $\left.\mathrm{R}_{\text {Global }}=0,27 ; \mathrm{p}=0,001\right)$. Galaxias maculatus y G. platei son las especies que mejor explican los hábitats de poza mientras que $T$. areolatus y $D$. camposensis son las que explican los hábitats rápidos (Tabla 3).

\section{DISCUSIÓN}

Los resultados muestran un uso diferenciado de los hábitats descritos por especie o grupo de especies, donde los galáxidos son más abundantes en hábitats de baja velocidad mientras que los bagres lo son en hábitats de alta velocidad. Por otro lado, sólo algunas especies muestran un uso diferenciado de hábitats por talla.

El uso de hábitat de los galáxidos estaría asociado a su historia de vida, incluyendo diadromía y reproducción. Galaxias maculatus es una especie diádroma que forma poblaciones migratorias y encerradas (Cussac et al. 2004), por lo que puede ser encontrada en una amplia diversidad de hábitats de río y zonas litorales de lago (Campos 1970; McDowall 1971a; Barriga et al. 2002; Cussac et al. 2004). Campos (1985) la describe como una especie típica de la zona potamal de los ríos. En el río San Pedro, los hábitats de poza, y en particular poza temporal, fueron los más utilizados por la especie. Los individuos de tallas pequeñas fueron encontrados frecuentemente tanto en los hábitats de playa del lago Riñihue como en el estuario, que podría indicar la existencia de al menos dos zonas de reproducción (Campos 1970; McDowall 1971a; Campos et al. 1974; McDowall 1976). Galaxias platei es una especie típica de ambientes lacustres donde ocupa principalmente ambientes profundos (McDowall 1971a; Bariga et al. 2002; Milano et al. 2002; Cussac et al. 2004; Habit et al. 2010), aunque también se ha encontrado en ambientes litorales someros (Milano 2006). En ambientes fluviales, Campos (1985) reportó adultos en la zona de ritrón y juveniles en la zona de potamon. En el río San Pedro, los hábitats de baja velocidad (pozas) fueron los más usados por esta especie. Sin embargo, las mayores tallas fueron capturadas tanto en pozas temporales (PT) como en rápidos (RS y $\mathrm{RP}$ ), estos ambientes rápidos con presencia de rocas (refugio) como también pozas con fondos blandos y aguas turbias han sido descritos como sitios de refugio ante depredadores (Milano 2002). Los individuos de menor tamaño fueron capturados con frecuencia en la playa del lago Riñihue, lo que haría de este un ambiente importante para la reproducción y reclutamiento de la especie.

Las especies del Género Aplochiton son diádromas, han sido asociadas a la presencia de tributarios de zonas litorales lacustres (Campos 1969), donde los juveniles serían especialmente abundantes (McDowall 1969, 1971b). Campos (1985) describe este género en la zona de ritrón de los ríos, donde los hábitats rápidos son más comunes. En el San Pedro se capturaron pocos individuos de Aplochiton taeniatus Jenyns, 1842, donde mayoritariamente individuos de menor talla ocuparon zonas litorales del lago Riñihue en las cercanías de tributarios, y los individuos de mayor talla junto con juveniles fueron capturados en hábitats rápidos en el río San Pedro. Brachygalaxias bullocki (Regan 1908) es una especie exclusiva de agua dulce y utiliza una gran diversidad de hábitats, como humedales o zonas inundadas laterales de grandes ríos (Campos 1985), arroyos, ríos y litoral de lagos (McDowall 1971a). Esta especie fue capturada sólo en la parte baja del río San Pedro, utilizando hábitats con presencia de vegetación sumergida (terrestre y/o acuática) independiente de la velocidad de la corriente (rápidos y pozas).

Los Aterinópsidos son especies típicas de lago y de la zona potamal de los ríos (Campos 1985). Odontesthes mauleanum (Steindachner, 1896) fue capturada en mayor abundancia en el lago Riñihue y estuvo casi ausente en el río. Las mayores tallas de Basilichthys australis Eigenmann 1927 fueron capturadas en la zona pelágica del lago, mientras que los juveniles fueron capturados en grandes cardúmenes en hábitats de poza del río, principalmente poza temporal.

Los juveniles de Diplomystes camposensis Arratia, 1987 ocuparon principalmente rápidos someros y profundos $(<$ $1 \mathrm{~m}$ ), donde las tallas más pequeñas (reclutas) hacen mayor uso de rápidos someros. En tanto, los hábitats de poza temporal y poza somera fueron menos utilizados por esta especie. Oyanedel (com. pers.), indica que los adultos de D. camposensis ocupan zonas profundas (no vadeables) en el cauce principal del río San Pedro. Trichomycterus areolatus fue capturado ocupando principalmente hábitats rápidos y en menor proporción pozas, como ha sido reportado en otros ríos como el Mapocho, Angostura, Andalién y Biobío (Arratia 1983; Manríquez et al. 1988; Campos et al. 1993 a,b; Ruiz 1993; Habit 2005). Los bagres T. areolatus y $D$. nahuelbutaensis (congenérica de $D$. 
camposensis) han sido descritas como especies bentónicas, que habitan principalmente los ambientes con velocidad de corriente alta, bien oxigenados, con hábitos diurnos y nocturnos, respectivamente (Arratia 1983; Campos et al. 1993 a,b; Ruiz 1993; Habit 2005). Los adultos ocupan zonas más profundas y con mayores velocidades, mientras que los juveniles ocupan pozas someras y pozas laterales (Vila et al. 1996; Habit 2005). Arratia (1983) y Manríquez (1988) reportan juveniles de T. areolatus en zonas de baja velocidad de la corriente y substrato relativamente fino en las orillas del cauce, tal como se aprecia en el río San Pedro, con gran cantidad de reclutas capturados en este tipo de hábitat. Destaca la ocurrencia de tallas mayores (con mayor variabilidad) en pozas profundas y playas en el San Pedro. Arratia (1983) describe que individuos más grandes prefieren profundidades hasta $40 \mathrm{~cm}$.

Percilia gillissi fue la especie de más amplia distribución, con altas abundancias en todos los tipos de hábitat ribereño. Fue colectada principalmente en rápidos y pozas del río y en menor proporción en pozas temporales. Las tallas menores ocurrieron en pozas temporales y playas, que pueden ser sus zonas de reclutamiento. Percilia irwini, su especie congenérica, ha sido descrita utilizando principalmente hábitats de pozas en el río Biobío (Campos et al. 1993a,b; Ruiz 1993), con velocidades hasta $0,5 \mathrm{~m} / \mathrm{s}$ (Habit \& Belk 2007). Pércidos de los géneros Percina y Etheostoma (en Norte América), similares al género Percilia han sido descritos ocupando todos los tipos de hábitats del río, prefiriendo rápidos con substrato de grava (Grossman \& Freeman 1987; Freeman 1995; Skyfield \& Grossman 2008). La gran plasticidad en el uso de hábitat de $P$. gillissi explica en parte su éxito poblacional en cuanto a abundancia. Tal como $P$. gillissi, juveniles de Percichthys trucha (Valenciennes 1833) ocupan todos los tipos de hábitat ribereño, con una tendencia hacia el uso de rápidos someros. En la cuenca del río Biobío, los juveniles de esta especie han sido observados en zonas de baja velocidad, con substrato grueso o vegetación (Ruiz et al. 1993). En estado adulto, habita en la columna de agua en zonas profundas de ríos y lagos (Campos et al. 1993 a,b; Ruiz et al. 1993), como fue observada en el lago Riñihue en este estudio.

Las mayores capturas de larvas de Geotria australis Gray, 1851 en el río San Pedro concuerdan con Jellyman \& Glova (2002) y Campos et al. (1993a), en que amocetes ocupan pozas temporales con lechos fangosos y pozas someras donde pueden enterrarse. En tanto, el estado de macroftalmia prefiere aguas con velocidades bajas (Jellyman \& Glova 2002). Aunque no se capturaron adultos de G. australis en el río San Pedro, las tallas más pequeñas en las playas del lago indican áreas de desove en la parte alta de la cuenca, como ha sido reportado en Nueva Zelanda (Jellyman \& Glova 2002).
Cheirodon australe fue más abundante en ambientes de pozas, asociado generalmente a vegetación acuática. Su presencia en rápidos fue escasa y se limitó individuos de tallas mayores $(3,8-4,0 \mathrm{~cm})$. En las cuencas del Biobío y Andalién, la especie congenérica $C$. galusdae ocupa las orillas de los ríos, con baja velocidad y profundidad del agua, y por lo general asociado a vegetación acuática y todo tipo de substrato (Campos et al. 1993a,b; Ruiz 1993). Cheirodon galusdae ha sido descrito en desove y en estados de larva y juvenil en el área litoral de lagos, y en zonas de ritrón, potamón y estuarial en las cuencas del Biobío y Andalién (Campos et al. 1993a,b; Ruiz 1993; Ruiz \& Marchant 2004). En el río San Pedro no se aprecia una diferencia en el tipo de hábitat en que destaquen larvas y juveniles, mientras que en la zona litoral del lago Riñihue no fueron capturados.

Cada especie analizada tiene una forma particular de relacionarse con su ambiente y presenta adaptaciones fisiológicas que le permiten ocupar diferentes tipos de hábitats a lo largo de su vida. La velocidad del agua es la variable determinante de estos hábitats y es la que mejor explica los patrones comunitarios. Una mayor oferta de los hábitats preferidos y necesarios para cada especie significará mejores condiciones para su éxito. Además, asociar cada especie o grupo de especies a determinados hábitats resulta de gran importancia para su conservación, puesto que entrega la información necesaria para la protección o restauración de los hábitats idóneos para el mantenimiento o recuperación de poblaciones amenazadas.

Próximos trabajos sobre uso de hábitat, además de incluir abundancia de peces, deberían incluir información más detallada sobre el uso específico (alimentación, reproducción, descanso) de cada hábitat durante la historia de vida de cada especie. En un sistema amenazado por perturbaciones antropogénicas, esta información permitirá desarrollar planes de manejo que aseguren una efectiva conservación de los peces nativos y la biodiversidad de los sistemas acuáticos dulceacuícolas.

\section{AGRADECIMIENTOS}

Este estudio fue financiado por COLBUN S.A., beca CONICYT D-21070133 de Alex García, beca CONICYT de Jorge González, proyecto CONICYT AT-2491002, proyecto CONICYT AT-24091095. El proyecto DIUC Semilla Patagonia 210.310.057-1SP financió la publicación y el proyecto Fondecyt 1110441 permitió finalizar el manuscrito. Especial agradecimiento a la Sra. Catalina Puga Valdés del Hotel Riñimapu, Alfredo y Marietta Burkhardt Gesswein del fundo Los Bajos, Sr. José Luis del Río del fundo Carmen del Trafún, a la Sra Cecilia y Raúl Carrere Iroume del fundo Champulli, Sr. Javier Araya Clericus del 
fundo El Maitén, a los Srs. Guillermo Perez-Cotapos y Jorge Álamos del fundo La Quinta, al Srs. Juan Carlos y Joaquín Barros del fundo Cun-Cun y al Sr. Eduardo Tamm del fundo Cuyincahuin.

\section{BIBLIOGRAFÍA}

Arratia, G. 1983. Preferencias de hábitat de peces siluriformes de aguas continentales de Chile (Fam. Diplomystidae y Trichomyctidae). Studies of Neotropical Fauna and Environment 18 (4): 217-237.

Barriga, J.P., Milano, D., Batín, M.A., Macchi, M.J. \& Cussac, V.E. 2002. Spatial and temporal distribution of landlocked Galaxias maculatus and Galaxias platei (Pisces, Galaxiidae) in a lake in South American Andes. New Zealand Journal of Marine and Freshwater Research 36: 349-363.

Campos, H. 1969. Reproducción de Aplochiton taeniatus Jenyns. Boletín del Museo Nacional de Historia Natural 29 (13): 207222.

CAmpos, H. 1970. Galaxias maculatus (Jenyns) en Chile, con especial referencia a su reproducción. Boletín del Museo Nacional de Historia Natural 31: 5-20.

Campos, H., Bucarey, E. \& Arenas, J.N. 1974. Estudios limnológicos del Lago Riñihue y Río Valdivia (Chile). Boletín de la Sociedad Biología Concepción 48: 47-67.

CAmpos, H. 1985. Distribution of fishes in the Andean rivers in the South of Chile. Archives of Hydrobiology 104 (2): 169-191.

Campos, H.H., Ruiz, V.H., Gavilán, J.F. \& Alay, F. 1993a. Peces del Río Biobío. In: Faranda F. \& Parra O., eds. Centro EULAChile, Universidad de Concepción, Concepción, Chile, 100 pp.

Campos, H.H., Gavilán, J.F., Alay, F. \& Ruiz, V. 1993b. Comunidad íctica de la hoya hidrográfica del Río Biobío. In: Faranda, F. \& Parra O., eds. Gestión de recursos hídricos de la cuenca del Río Biobío y del área marina costera adyacente.Centro EULAChile, Universidad de Concepción, Concepción, Chile. pp. 249-278.

Clarke, K.R. \& Gorley, R.N. 2005. Primer-E version 6, user manual. Primer-E Ltd., Plymouth Marine Laboratory, Plymouth, UK.

Clifford, N.J., Harmar, O.P., Harvey, G. \& Petts, G.E. 2006. Physical habitat, eco-hydraulics and river design: a review and re-evaluation of some popular concepts and methods. Aquatic Conservation: Marine and Freshwater Ecosystems 16 (4): 389408.

Cussac, V.E., Ortubay, S., Iglesias, G., Milano, D., Lattuca, M.E., Barriga, J.P., Battini, M.A. \& Gross, M. 2004. The distribution of South American galaxiid fishes: the role of biological traits and post-glacial history. Journal of Biogeography 31: 103-121.

Freeman, M.C. \& Grossman, G.D. 1993. Effects of habitat availability on dispersion of a stream cyprinid. Environmental Biology of Fishes 37 (2): 121-130.

Freeman, M. 1995. Movements by two small fishes in a large stream. Copeia 1995 (2): 361-367.

García, A., Jorde, K., Habit, E., CaAmaño, D. \& Parra, O. 2011. Downstream environmental effects of dam operations: changes in habitat quality for native fish species. River Research and
Applications.

Grossman, G.D. \& Freeman, M.C. 1987. Microhabitat use in a stream fish assemblage. Journal of Zoology 212: 151-176.

НАвіт, E. 2005. Aspectos de la biología y hábitat de un pez endémico de Chile en peligro de extinción (Diplomystes nahuelbutaensis Arratia 1987). Interciencia 30: 1-10.

Habit, E. \& Belk, M.C. 2007. Threatened fishes of the world: Percilia irwini (Eigenmann 1927)(Perciliidae). Environmental Biology of Fishes 78 (3): 213-214.

Habit, E., Piedra, P., Ruzzante, D.E., Walde, S.J., Belk, M.C., Cussac, V.E., González, J. \& Colin, N. 2010. Changes in the distribution of native fishes in response to introduced species and other anthropogenic effects. Global Ecology and Biogeography 19 (5): 697-710.

Hawkins, C.P., Kershner, J.L., Bisson, P.A., Bryant, M.D., Decker, L.M., Gregory, S.V., McCullough, D.A., Overton, C.K., Reeves, G.H., Steedman, R.J. \& Young, M.K. 1993. A hierarchical approach to classifying stream habitat features. Fisheries 18 (6): 3-12.

Jellyman, D.J. \& Glova, G.J. 2002. Habitat use by juvenile lampreys in a large New Zealand river. New Zealand Journal of Marine and Freshwater Research 36: 503-510.

JowetT, I. 1993. A method for objectively identifying pool, run, and riffle habitats from physical measurements. New Zealand Journal of Marine and Freshwater Research 27 (2): 241-248.

Manríquez, A., Huaquin, L., Arellano, M. \& Arratia, G. 1988. Aspectos Reproductivos de Trichomycterus areolatus Valenciennes, 1846 (Pisces: Teleostei: Siluriformes) en Río Angostura Chile. Studies on Neotropical Fauna and Environment 23 (2): 89-102.

McDowall, R.M. 1969. A juvenile of Aplochiton Jenyns. Copeia 3: 631-632.

McDowall, R.M. 1971a. The Galaxiid fishes of South America. Zoological Journal of the Linnean Society 50 (1): 33-73.

McDowall, R.M. 1971b. Fishes of the family Aplochitonidae. Journal of the Royal Society of New Zealand 1 (1): 31-52.

McDowall, R.M. 1976. The taxonomic status of the Galaxias populations in the Rio Calle Calle, Chile (Pisces: Galaxiidae). Studies on Neotropical Fauna and Environment 11: 173-177.

Milano, D., Cussac, V.E., Macchi, P.J., Ruzzante, D.E., Alonso, M.F., Vigliano, P.H. \& Denegri, M. 2002. Predator associated morphology in Galaxias platei in Patagonian lakes. Journal of Fish Biology 61 (1): 138-156.

Milano D., D. Ruzzante, V. Cussac, P. Macchi, R. Ferriz, J. Barriga, J. Aigo, M. Lattuca \& S. Walde. 2006. Latitudinal and ecological correlates of morphological variation in Galaxias platei (Pisces, Galaxiidae) in Patagonia. Biological Journal of the Linnean Society 87: 69-82.

Parasiewicz, P. 2001. MesoHABSIM: A concept for application of instream flow models in river restoration planning. Fisheries 26 (9): 6-13.

Penaluna, B.E., Arismendi, I. \& Soto, D. 2009. Evidence of Interactive Segregation between Introduced Trout and Native Fishes in Northern Patagonian Rivers, Chile. Transactions of the American Fisheries Society 138: 839-845.

RuIz, V.H. 1993. Ictiofauna del Río Andalién (Concepción, Chile). Gayana Zoología 57 (2): 109-278.

Ruiz, V.H., López, M.T., Moyano, H.I. \& Marchant, M. 1993. Ichthyology of the high Biobío River system: some taxonomical, alimentary, reproductive and ecological aspects 
and a discussion on the river basin. Gayana Zoología 57 (1): 77-88.

RuIZ, V.H. \& MARChant, M. 2004. Ictiofauna de aguas continentales. Facultad de Ciencias Naturales y Oceanográficas, departamento de Zoología, Universidad de Concepción, Concepción, Chile $356 \mathrm{pp}$.

Skyfield, J.P. \& Grossman, G.D. 2008. Microhabitat use, movements and abundance of gilt darters (Percina evides) in southern Appalachian (USA) streams. Ecology of Freshwater Fish 17 (2): 219-230.

Thomasson, K. 1963. Araucanian lakes. Acta Phytogeographica Sueca 47: 1-139

Tickner, D., Armitage, P.D., Bickerton, M.A. \& Hall, K.A. 2000. Assessing stream quality using information on mesohabitat distribution and character. Aquatic Conservation: Marine and Freshwater Ecosystems 10: 179-196.

Vannote, R. L., G. W. Minshall, K. W. Cummings, J. R. Sedell,
\& Cushing, C. E. 1980. The River Continuum Concept. Canadian Journal of Fisheries and Aquatic Sciences 37: 130137.

Vargas, P.V., Arismendi, I., Lara, G., Millar, J. \& Peredo, S. 2010. Evidencia de solapamiento de micro-hábitat entre juveniles del salmón introducido Oncorhynchus tshawystscha y el pez nativo Trichomycterus areolatus en el río Allipén, Chile. Revista de Biología Marina y Oceanografía 45 (2): 285-292.

Vila, I., Contreras, M. \& Fuentes, L. 1996. Reproduction of Diplomystes nahuelbutaensis Arratia 1987 (Pisces: Diplomystidae). Gayana Oceanología 4 (2): 129-137.

Werner, E.E. \& HaL, D.J. 1976. Niche shifts in sunfishes: experimental evidence and significance. Science (New York, N.Y.) 191 (4225): 404-406.

Werner, E.E. \& Gill, J.F. 1984. The ontogenetic niche and species interactions in size-structured populations. Annual Review of Ecology, Evolution, and Systematics 15: 393-425.

Recibido: 02.01.12

Aceptado: 27.02.12 